\title{
The importance of molecular friction
}

\section{in a soft polymer-nanotube nanocomposite}

Tao Wang, Alan B. Dalton and Joseph L. Keddie*

Department of Physics and Surrey Materials Institute, University of Surrey, Guildford, Surrey GU2 7XH, United Kingdom

Running title: Molecular friction in soft nanocomposites

*Corresponding author: Joseph L. Keddie (j.keddie@ surrey.ac.uk) 
Published in Macromolecules (2008) 41(20):7656-7661

ABSTRACT: The effects of physisorbed polymer molecules on carbon nanotubes dispersed in a soft polymer matrix on the resulting mechanical strength of the nanocomposite are reported. From measurements of the large-strain deformation of the nanocomposites, the shear strength, $\tau$, of the nanotube/matrix interface was determined as a function of the interfacial polymer chain length and the chain density, $\Sigma$. The results show that the value of $\tau$ (per chain) increases with increasing chain length. $\tau$ likewise increases with $\Sigma$, but then levels off above a critical value. These results are explained by the molecular friction of the adsorbed polymer chains sliding along the rubbery polymer matrix. The results can be used to guide the interfacial design of polymer nanocomposites to obtain ultimate macroscopic mechanical control. In particular, the monomeric friction coefficient, $\xi_{1}$, could be used to adjust the macroscopic properties of this type of nanocomposite. 


\section{Introduction}

It is well accepted in the literature on polymer composites that the interface between a fiber and the continuous matrix has a profound effect on the macroscopic mechanical performance. ${ }^{1}$ The more recent literature on polymer nanocomposites containing high aspect-ratio nanofillers (e.g. platelets, ${ }^{2}$ carbon nanotubes, ${ }^{3}$ and fibers ${ }^{4}$ ) has been built upon the models of "classic" uniaxial composites. The models predict that the fracture mechanism and the ultimate strength and toughness of this type of nanocomposite depend on two key parameters: the filler length $(L)$ and the interfacial shear strength $(\tau)$ between the matrix and the filler (representing the stress at failure). A nanofiller will break during deformation of the nanocomposite only when the stress transferred to it is larger than its fracture strength. The stress transferred from the matrix depends on both $L$ and $\tau$. If the transferred stress does not exceed the filler's fracture strength, then the filler will pull out from the matrix when the stress level exceeds $\tau$. The two key parameters can be adjusted by chemical or physical means to induce either a filler fracture mechanism or a filler pull-out mechanism. They therefore provide a tool for the design of the mechanical performance of nanocomposites.

There are a few examples in the literature that illustrate the importance of the interfaces between nanofillers and the matrix. For instance, spherical nanoparticles in glassy polymers ${ }^{5}$ are very effective in toughening the nanocomposite when there is high interfacial adhesion, obtained when the testing temperature is above the glass transition temperature $\left(T_{g}\right)$ of the polymer matrix. In a second example, when polymers are grafted onto multi-walled carbon nanotubes (MWNTs) to impart miscibility with the matrix, the resulting efficient load transfer from the matrix to the nanotubes increases the mechanical properties, such as modulus, strength, ultimate strain and toughness. ${ }^{6}$ Additionally, a more effective interfacial stress transfer and a pronounced mechanical reinforcement have been achieved by using carbon nanotubes with a high density of polymer chains grafted onto them. ${ }^{7}$

Although there is a general consensus that carbon nanotubes require surface treatments or chemical functionalisation to achieve good dispersability and compatibility with the matrix, ${ }^{8}$ the effects of the 


\section{Published in Macromolecules (2008) 41(20):7656-7661}

interfacial composition and structure on the resultant interfacial strength lack quantitative experimental studies. There has been greater emphasis on chemical modifications of the filler/matrix interfaces (e.g. covalent bonding) rather than on physical mechanisms, as will be presented here. A better understanding of how to control $\tau$ may enable the bottom-up design of nanocomposites with desired properties.

This present work is partially inspired by previous studies of how polymer chains at interfaces influence fracture, adhesion, and friction. Kramer and co-workers systematically investigated the interfacial fracture strength of glassy polymer/polymer interfaces that are reinforced with miscible diblock copolymers. ${ }^{9-11}$ They measured the interfacial fracture toughness using the double cantilever beam method and studied its dependence on the copolymer degree of polymerisation, $N$, and interfacial chain density, $\Sigma$ (expressed as chains $\mathrm{nm}^{-2}$ ). They found that the interfacial failure occurs by copolymer chain pull-out from the homopolymer when the $N$ of each block is less than the value at entanglement $\left(N_{e}\right)$. If $N$ is above $N_{e}$ but with a low $\Sigma$, the failure is by chain scission, as the entangled chains break under stress rather than pull-out from the matrix.

The adhesion strength at interfaces between elastomers and polymer brushes grafted on solid surfaces is similarly affected by chain entanglements. The energy of adhesion increases with both the $\Sigma$ and $N$ of the brush. ${ }^{12}$ Furthermore, Brown's pioneering experiments ${ }^{13}$ on the sliding friction between elastomers and grafted polymer chains on solid, planar supports found a strong dependence on the interfacial chain thickness. Later, other systematic studies ${ }^{14,15}$ showed that the friction stress between a tethered brush and an elastomer increases with $\Sigma$.

This present work concerns nanocomposites made from multi-wall carbon nanotubes dispersed in a soft polymer matrix using a waterborne colloidal process. ${ }^{16,17}$ To make these nanocomposites, the nanotubes are dispersed in water through the physisorption of a hydrophilic poly(vinyl pyrrolidone) (PVP). In the dry nanocomposite, PVP is then located mainly at the interface between the nanotube and the rubbery matrix. Our nanocomposite system can therefore be broadly described as a rubbery polymer in contact with polymer chains adsorbed on a curved surface of solid carbon. The macroscopic 


\section{Published in Macromolecules (2008) 41(20):7656-7661}

properties of the nanocomposite are determined, in part, by $\tau$. We report here a systematic investigation of the effects of the $\Sigma$ and $N$ of the interfacial PVP on the observed macroscopic mechanical properties of the nanocomposites. The results are analyzed through calculations of $\tau$. When interpreting the results lessons are learned and applied from the literature on the friction of rubbers sliding along polymer brushes on solid surfaces.

\section{Experimental Details}

\section{MWNT-PVP Aqueous Dispersions}

MWNTs (Nanocyl, Belgium) are reported by the manufacturer to have a purity level of $95 \%$ and were used as received. TEM analysis, shown in the supporting information in our previous work ${ }^{17}$ revealed that the average length, $L$, of the MWNTs is $1,500 \mathrm{~nm}$, and the average diameter, $D$, is $20 \mathrm{~nm}$. The average inner diameter, $D_{i}$, is $8 \mathrm{~nm}$. The MWNTs were dispersed in solutions of poly(vinyl pyrrolidone) (PVP, Sigma-Aldrich) in water. For all experiments, an initial MWNT concentration was fixed at 0.1 wt.\% in water; various PVP concentrations were added, as desired, to achieve a range of interfacial chain densities. Various PVP molecular weight, $M_{w}$, fractions, namely 1 (1.2), 10 (2.7), 29 (5.2), 360 (5.4) and $1570(5.3) \mathrm{kg} \mathrm{mol}^{-1}$ (where the polydispersity index, $M_{w} / M_{n}$, is given in parentheses) were used to investigate the effect of chain length. The average degree of polymerization, $\bar{N}$, of the PVP thereby ranged from 9 to 14,144 . When investigating the effect of chain density on mechanical properties, PVP with $M_{w}$ of $1570 \mathrm{k}$ was used while the PVP: MWNT ratio was varied.

MWNTs were added to aqueous PVP solutions, and the mixtures were sonicated for $10 \mathrm{~min}$. using a sonic tip with output power of 20W (Branson Sonifier 150, Branson Ultrasonics Corp, Danbury, CT) followed by mild sonication for $30 \mathrm{~min}$. in a sonic bath. During both sonications, the dispersions were chilled by immersion in ice water. Then the dispersions were centrifuged for $30 \mathrm{~min}$. at $4400 \mathrm{rpm}$, and approximately $90 \%$ of the dispersion was collected as a supernatant of nanotubes dispersed in water. The supernatant collected after centrifuging at $4400 \mathrm{rpm}$ contains MWNTs with adsorbed PVP chains 
on them and with free PVP chains in the solution. TEM analysis shows that MWNTs were, for the most part, homogeneously dispersed and de-bundled in the supernatant.

\section{PVP Chain Density Determination}

To determine the amount of PVP adsorbed on the MWNTs, the supernatants were further centrifuged at 50,000 rpm for two hr. The MWNTs with adsorbed PVP chains were isolated at the bottom of the centrifugation tubes, and the free PVP chains in water were isolated at the top. The PVP solution was decanted off, and the MWNTs with adsorbed PVP were collected and dried. The weight ratio, $w$, of adsorbed PVP to MWNTs was determined with thermogravimetric measurements in nitrogen (Q500, TA Instruments, New Castle, USA). In this analysis, dried solutions of PVP adsorbed on MWNTs were heated to a temperature of $800{ }^{\circ} \mathrm{C}$, which is well above the temperature for the complete decomposition of PVP $\left(600{ }^{\circ} \mathrm{C}\right)$, as reported previously. ${ }^{17}$ The mass loss attributed to the PVP decomposition was compared to the total mass and used to find $w$. The number ratio, $m$, of PVP:MWNT (i.e. number of PVP chains per nanotube) was then calculated for each dispersion, by modellng the MWNTs as cylinders with a mass density, $\rho$, of $2.15 \times 10^{6} \mathrm{~g} \mathrm{~m}^{-3(18)}$ in the expression:

$$
m=\frac{\pi\left(\frac{D}{2}\right)^{2} L \rho w}{M_{w}} N_{A}=\frac{\pi D^{2} L \rho w N_{A}}{4 M_{w}}
$$

where $N_{A}$ is Avogadro's number. The areal number density of adsorbed PVP chains, $\Sigma$ (chains $\mathrm{nm}^{-2}$ ), was then found by considering the surface area of the outer wall, $A_{w}$, of the MWNTs, expressed as

$$
\sum=\frac{m}{A_{w}}=\frac{m}{\pi D L}
$$

\section{Nanocomposite Preparation and Measurement}

To prepare nanocomposites, the free PVP was not removed from the dispersion, so as to maintain equilibrium between the adsorption and desorption of the dispersant on the nanotube surface. The 
MWNT dispersions were blended while stirring into a colloidal dispersion of poly(butyl acrylate-coacrylic acid) (poly(BuA-co-AA)), prepared by emulsion polymerization, as described previously ${ }^{16}$ (Figure 1a). The polymer is lightly crosslinked so that there is a gel fraction of $30 \%$. The average particle size is $120 \mathrm{~nm}$, and the $T_{\mathrm{g}}$ of the copolymer is $-50{ }^{\circ} \mathrm{C}$. The surfaces of the particles are enriched in acrylic acid groups.

In all experiments, the volume fraction of MWNTs, $\phi_{N T}$, calculated on the polymer in the dry films, was $7 \times 10^{-5}$. (The volume of the adsorbed PVP is not included in the calculation of the volume of the MWNTs.) To check the mechanical effects of this free PVP which is not removed from the nanocomposite, the same amount of free PVP solution (i.e. the supernatants of the solutions centrifuged at 50,000 rpm for two hr.) were added to the latex to prepare latex/PVP dispersions for control experiments.

All colloidal dispersions were cast in poly(tetrafluoroethylene) molds and allowed to dry at room temperature for seven days. Interdiffusion occurs readily across the $\mathrm{P}(\mathrm{BuA}-\mathrm{co}-\mathrm{AA})$ particle/particle boundaries $^{19}$ because of the very low $T_{\mathrm{g}}$ and low level of crosslinking; particle coalescence creates a homogeneous matrix. ${ }^{20}$ Tensile specimens $(10 \mathrm{~mm}$ x $1 \mathrm{~mm}$ x $1 \mathrm{~mm})$ were cut from the dried nanocomposite layers. Tensile stress-strain measurements were obtained on an apparatus (Texture Analyzer, Stable Micro Systems Ltd., Goldaming, UK) with a constant strain velocity of $5 \mathrm{~mm} \mathrm{~s}^{-1}$, corresponding to an initial strain rate of $c a .0 .5 \mathrm{~Hz}$. Three or more replicate experiments were performed for each $N$ and $\Sigma$ under investigation, and average values are reported here.

\section{Interfacial Strength Determination}

To study the interfacial strength, $\tau$, between a single nanotube and the matrix, a suitable technique must be devised to measure a single interface. AFM-assisted nanomechanics experiments have been employed elsewhere to measure the interfacial adhesion between single nanotubes and the matrix. ${ }^{21}$ In this work, $\tau$ is deduced from large-scale deformation of the nanocomposite, using the working hypothesis outlined below. 


\section{Published in Macromolecules (2008) 41(20):7656-7661}

In classical models of conventional uni-axial fiber-reinforced composites that fail by fiber pull-out, ${ }^{4}$ the macroscopic strength is proportional to the interfacial shear strength between the fiber and the matrix. This model has been extended to nanocomposites and applied to nano-sized fillers, such as platelets. $^{2}$ For nanocomposites filled with nanotubes, Coleman et $a l^{3}$ showed how the interfacial stress transferred to the nanotube scales with $L$. A critical nanotube length, represented as $L_{c}$, is predicted at which enough stress is transferred to the nanotube to break it. This length is expressed as

$$
L_{c}=\frac{\sigma_{N T} D}{2 \tau}\left[1-\frac{D_{i}{ }^{2}}{D^{2}}\right]
$$

where $\sigma_{\mathrm{NT}}$ is the nanotube strength (given in the literature as $\left.50 \mathrm{GPa}\right)^{3}$ and $\tau$ is the interfacial strength between the nanotube and matrix.

Composite materials with a weak interface have been found to have experimental values of $\tau$ near 10 $\mathrm{MPa}^{[21]}$ However, both computer simulation ${ }^{22}$ and experimental work ${ }^{23}$ have reported $\tau$ values of around $100 \mathrm{MPa}$ when there is strong covalent bonding at the interface. So as $\tau$ varies between 10 and $100 \mathrm{MPa}, L_{c}$ ranges between approximately 4 and $40 \mu \mathrm{m}$. Thus, in our experiments in which $L=1.5$ $\mu \mathrm{m}$, it is valid to assume that $L<L_{c}$ even if $\tau$ is considered to have a value at the upper limit.

In the case where $L<L_{c}$, the stress transferred to the nanotubes is not sufficient to break them, and the failure of the nanocomposite thus occurs by the nanotube pulling out from the matrix. In this pull-out process, the failure is controlled by $\tau$. The composite strength $\sigma_{\mathrm{c}}$ can be obtained from a knowledge of the matrix strength $\sigma_{\mathrm{m}}$ using the standard expression: $:^{3,4}$

$$
\sigma_{c}=\left(\tau \frac{L}{D}-\sigma_{m}\right) \phi_{N T}+\sigma_{m}
$$

Measurements of $\sigma_{\mathrm{c}}$, coupled with measurements of the other parameters, thereby allowed us to calculate $\tau$ by inversion of Eq. 4 .

We note that Eq. 4 was derived through the extension of a model for uniaxial fiber-reinforced composites. The carbon nanotubes in our nanocomposites are not expected initially to be aligned 
Published in Macromolecules (2008) 41(20):7656-7661

parallel to each other. However, in the large strain regime, in which our experiments are conducted, nanotubes in a polymer matrix have been shown elsewhere ${ }^{24,25}$ to align in the direction of a tensile stress. Hence, we proceed in applying Eq. 4 after noting the underlying assumption in the analysis and treating it as a working hypothesis.

\section{Results and Discussion}

There are several factors that can influence the mechanical properties of nanocomposites: matrix morphology, the extent of nanotube dispersion (or bundling), the interface type and structure, and the nanotube orientation. The matrix $\mathrm{P}(\mathrm{BuA}-\mathrm{co}-\mathrm{AA})$ here is in a rubbery state and unable to crystallize. Our calorimetry measurements clearly showed that the nanocomposites with the presence of MWNTs have no crystallization and the matrix is still amorphous. As illustrated in our previous work ${ }^{17}$, MWNTs are de-bundled in the dispersion, and all of the nanocomposites show a similar uniform dispersion of MWNTs, regardless of the PVP chain lengths. The MWNTs in the matrix are randomly dispersed with similar aspect ratios, and there is no preferential orientation. 



High $\square$ and Low $N$

Figure 1. Schematic diagram of the preparation of the nanocomposites and the resulting interfaces. a) An illustration of PVP chains adsorbed on MWNTs dispersed in water blended with the colloidal $\mathrm{P}(\mathrm{BuA}-\mathrm{co}-\mathrm{AA})$ particles. $\mathrm{b})$ Interfacial structure for a clean interface, which is not possible to achieve in the colloidal route of processing. c) Illustration of PVP chains with a high $\bar{N}$ at a MWNT/matrix interface at low $\Sigma$. d) Illustration of PVP chains with a low $\bar{N}$ at a MWNT/matrix interface at high $\Sigma$. There is limited miscibility between the PVP chains and the matrix. 
The effects of the incorporation of the free PVP chains in the preparation procedure were investigated through large-strain tensile deformation measurements (Fig. S1). The concentration of the free PVP chains had no significant impact on the strentgh or toughness of the $\mathrm{P}(\mathrm{BuA}-\mathrm{co}-\mathrm{AA})$ films. The primary differences in the nanocomposites under investigation are the $\bar{N}$ and $\Sigma$ of the PVP dispersant chains, which are primarily localised at the interface between the MWNTs and the P(BuA-co-AA) matrix.

The $\mathrm{P}(\mathrm{BuA}-\mathrm{co}-\mathrm{AA})$ particle surface is enriched in $\mathrm{AA}$, and $\mathrm{PVP}$ is miscible with $\mathrm{AA}^{26}$. Plasticisation of the acrylic acid-rich chains by water might facilitate their extension and interpenetration with the PVP chains. The schemes in Fig. 1c and d illustrate the PVP chains at the matrix/MWNT interfaces for two different $\bar{N}$ and $\Sigma$ values.

Large-strain tensile deformation measurements revealed that the maximum strength of the $\mathrm{P}(\mathrm{BuA}-\mathrm{co}-$ AA) films is $0.14 \mathrm{MPa}$, which defines the value of $\sigma_{\mathrm{m}}$ for subsequent calculations. This value of $\sigma_{\mathrm{m}}$ was obtained from the maximum stress achieved on the stress-strain curve, as illustrated in Fig. 2a. This same figure also compares the deformation of the polymer films to that of the nanocomposites having a constant polymer chain length $(\bar{N}=14,144)$ but varying $\sum$. The figure illustrates that with a higher $\Sigma$, the nanocomposite strength, $\sigma_{c}$ (defined as the maximum stress obtained during large-strain deformation), increases, and also greater strains are achieved before failure. Measurements of $\sigma_{c}$ are used to calculate values of $\tau$ through inversion of Eq. 4. The dependence of $\tau$ on $\Sigma$ is shown in Fig. $2 b$. A linear relation between $\tau$ and $\Sigma$ is observed up to $\Sigma=0.0045$ chains $/ \mathrm{nm}^{2}$. Thereafter, $\tau$ remains constant at a plateau value of $20 \mathrm{MPa}$. With more polymer chains at the interface, the interaction with matrix chain is increased and consequently the interfacial strength is raised. Above a critical chain density, however, the effect of $\Sigma$ levels off. 

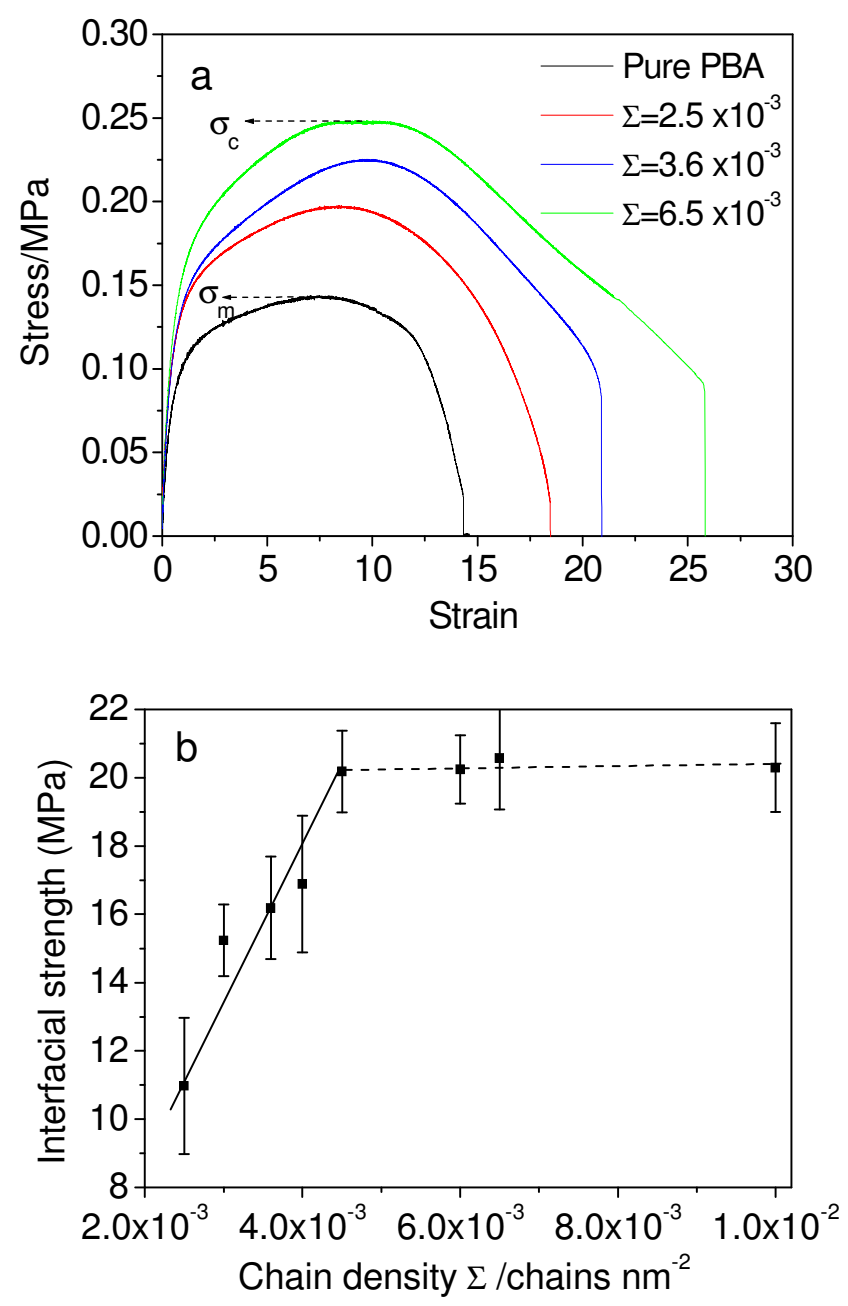

Figure 2. a) Tensile deformation of pure $\mathrm{P}(\mathrm{BuA}-\mathrm{co}-\mathrm{AA})$ and the nanocomposites with the same average chain length $(\bar{N}=14,144)$ but different $\Sigma\left(\right.$ chains $\left.\mathrm{nm}^{-2}\right)$ at the interface, as indicated in the legend. The engineering stress is plotted against the strain (change in length over initial length). b) The dependence of $\tau$ on $\Sigma$ for these same nanocomposites. The solid line is the best linear fit to the data.

It is worthwhile noting that other studies ${ }^{14,15}$ of macro-scale sliding at planar interfaces found that the friction stress likewise increased linearly with grafting density until reaching a critical value, above which the stress reached a plateau. Bureau and Léger ${ }^{14}$ have proposed that at high $\Sigma$, the sliding friction is set by the rheology of the thin layer of interfacial chains. This idea provides a good explanation for the plateau of $\tau$ found for high $\Sigma$ in Fig. 2b. We also note that a similar dependence on interfacial chain 


\section{Published in Macromolecules (2008) 41(20):7656-7661}

density was found in the interaction of diblock copolymer brushes at the interfaces in immiscible polymer blends. ${ }^{9-11}$ When the $\Sigma$ became sufficiently large, the interfacial strengths were found to level off at a constant value. In Brown's experiments, ${ }^{13}$ sliding friction was increased by thin brush layers (i.e. low $\Sigma$ ) but liquid-like behavior was found for thicker layers.

Fig. 3a shows the large-strain tensile deformation of the nanocomposites with varying PVP chain length. Experimentally, it was not possible via the physisorption process to hold $\Sigma$ perfectly constant while varying $\bar{N}$, so there is some variation in both parameters. The quantitative dependence of $\tau$ on $\bar{N}$ was then determined experimentally through analysis of the data. $\tau$ was normalized by $\Sigma$ so as to represent the average force provided per individual chain during the pull-out process. Underlying this calculation is an assumption that $\tau$ is negligible when there are no PVP chains at the interface between the nanotube and matrix. Experimentally, it is not possible to create such an interface, because the nanotubes require a dispersant to be suspended in water. However, surfactants are small molecules and hence will not entangle with the matrix nor create a high friction. Previously ${ }^{17}$ we reported that $\sigma_{\mathrm{c}}$ for nanocomposites prepared with surfactants is just slightly higher than $\sigma_{\mathrm{m}}$, showing that $\tau \approx 0$ when the matrix is not sliding along polymer chains adsorbed at the interfaces. Hence, $F_{\text {frict }} \approx \tau \tau \Sigma$.

Fig. $3 b$ presents experimental measurements of $F_{\text {frict }}$ obtained by varying $\bar{N} . F_{\text {frict }}$ is shown here to scale with $\bar{N}$ to a power of 1 (within the experimental uncertainty). On linear axes, the extrapolation of $\tau / \Sigma$ to $\bar{N}=0$ is very small $(<2 \mathrm{pN}) . F_{\text {frict }}$ is three orders of magnitude higher (on the order of $\mathrm{nN}$ ) when $\bar{N}=14,144$. This range of values of $F_{\text {frict }}$ is comparable to those found elsewhere ${ }^{9,27}$ for the force per chain when the pull-out of diblock copolymer chains occurred at an immiscible polymer/polymer interface. Longer chains, above their $N_{\mathrm{e}}$, were found to create entanglements on either side of the interface, which resist their easy pull-out. In this case, the failure occurs by chain scission. Values in the range of 1 to $3 \mathrm{nN}$ have been reported experimentally ${ }^{9,27,28}$ as being required to break a C-C bond in a chain scission process. The range of $F_{\text {frict }}$ values found in the data in Fig. $3 \mathrm{~b}$ (except for the largest $N$ ) 
is thus lower than what is expected for a chain scission mechanism but is in line with the expected force of friction in a chain sliding mechanism, as will be shown hereafter.
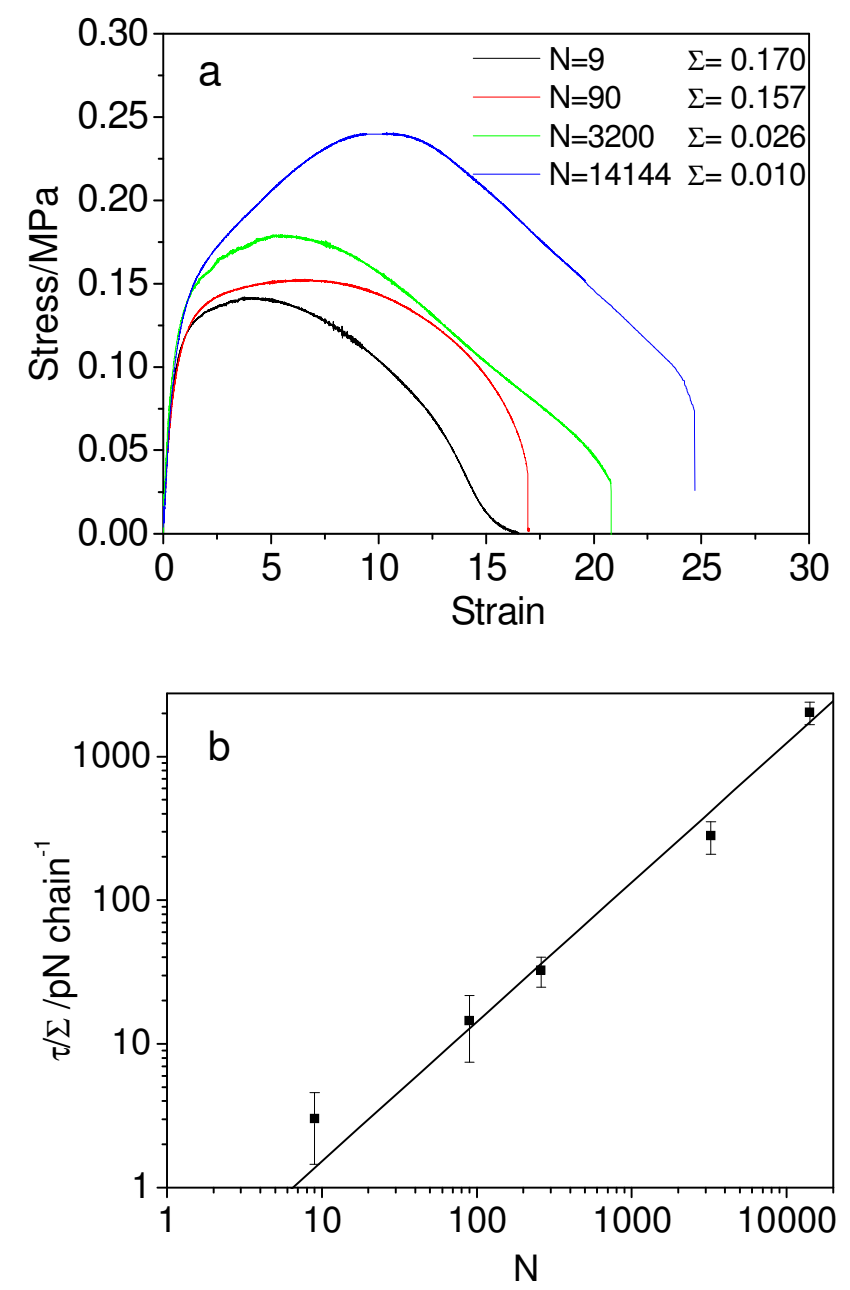

Figure 3. a) Tensile deformation of the nanocomposites with various polymer chain lengths (measured through $\bar{N}$ ) at the nanotube/matrix interface, as indicated in the legend. b) The dependence of the sliding force per interfacial chain on $\bar{N}$. The solid line is the linear fit with a slope of $1.00 \pm 0.06$

In order for the PVP chains to sustain such relatively high forces, they must be attached strongly to the MWNTs. Molecular dynamics simulations using empirical force fields ${ }^{29}$ offer some insight into the expected interaction energies solely due to van der Waals interactions between polymers and CNTs. The energy depends on the temperature and the nanotube diameter. The simulation for poly(styrene) (PS) on a nanotube with a $20 \mathrm{~nm}$ diameter (the same size as used in our work) finds an interaction 
energy of approximately $250 \mathrm{~kJ} / \mathrm{mole}$. Note that PS has some structural similarities to PVP. This energy level is below the covalent bond energy of a single C-C bond, such as is found in the backbone of PVP, with a value of $360 \mathrm{~kJ} /$ mole. $^{30}$ Hence, it is expected that the chains can support significant stress without pulling off of the MWNT surface but not enough stress to cause chain scission.

For an elastomer sliding along a polymer brush, the friction force acting on a chain, $F_{\text {frict }}$, depends on the velocity, $V$, of the sliding. ${ }^{31,32}$ Below a certain velocity $V_{r}$, the whole chain of the brush can relax in the elastomer network. ${ }^{14}$ This velocity is given as

$$
V_{r}=D_{e} / \tau_{\text {arm }}(N)
$$

with $D_{e} \approx a \sqrt{N_{e}}$ being the mesh size of the rubber network and $\tau_{\text {arm }}(N)$ being the Rouse relaxation time of a chain with $N$ monomers. The monomer size, $a$, can be estimated to be $0.5 \mathrm{~nm} . N_{e}$, the number of repeat units between entanglements, is estimated to be 100 for the $\mathrm{P}(\mathrm{BuA}-\mathrm{co}-\mathrm{AA})$ matrix.

Above $V_{r}$, the head of the chain is stretched in a straight tube of diameter $D_{e}$ and only a tail of $q$ monomers has time to relax, coil and penetrate into the rubber network. ${ }^{15}$ When the velocity increases, the coil will shrink and the stretched chains will grow until $q$ is less than $N_{e}$.

In the velocity regime where $V>V_{s}$, the whole chain has no time to relax, and it is mainly stretched. $V_{\text {s }}$ can be written ${ }^{14}$ as

$$
V_{s}=\frac{D_{e}}{\tau_{m} N N_{e}}
$$

where $\tau_{1}$ is the monomeric relaxation time expressed as $\tau_{m}=\xi_{1} a^{2} /\left(3 \pi^{2} k T\right)$. Here, $k$ is the Boltzmann constant, $T$ is the absolute temperature $(300 \mathrm{~K})$, and $\xi_{l}$ is the monomeric friction coefficient, which is the frictional force per monomer unit per unit velocity moving through a medium consisting of other polymer molecules. $\xi_{1}$ is a function of both the geometry of the repeat unit and its chemistry. ${ }^{33}$ The $\xi_{1}$ for rubbery polymers is typically on the order of $50 \mathrm{nNs} \mathrm{m}^{-1}$ at room temperature. ${ }^{34}$ The corresponding monomer relaxation time, $\tau_{\mathrm{m}}$, will be on the order of $10^{-7} \mathrm{~s}$. 
For PVP chains with $\bar{N}=3,243$ and 14,144, we estimate, using Eq. 6, that $V_{\mathrm{s}}$ is 0.2 and $0.04 \mu \mathrm{m} / \mathrm{sec}$, respectively. The local sliding velocity $V$ at the nanotube/matrix interface is taken to be $0.75 \mu \mathrm{m} / \mathrm{sec}$ (as estimated from the product of $L$ and the initial strain rate: $1.5 \mu \mathrm{m} \times 0.5 \mathrm{~Hz}$ ). Thus, for $\bar{N}=3,243$ and 14,144 , the sliding velocity $V$ is found to be larger than $V_{s}$.

When $\mathrm{V}>V_{\mathrm{s}}$, the sliding friction is attributed to the Rouse friction with the rubber network. The frictional force per chain is then linearly proportional to $V$, such that

$$
F_{\text {frict }}=\xi_{1} N V
$$

This equation is applicable for the two higher $\bar{N}$ and can be used to calculate the monomeric friction coefficient of PVP with the $\mathrm{P}(\mathrm{BuA}-\mathrm{co}-\mathrm{AA})$ matrix. We find $\xi_{l}$ to be on the order of $100 \mathrm{nN} \mathrm{s} \mathrm{m}^{-1}$ in our nanocomposite. This value is reasonable, when compared to values obtained for other polymers with polar repeat units, such as poly(vinyl chloride) $\left(30 \mathrm{nN} \mathrm{sm}^{-1}\right.$ at $\left.125{ }^{\circ} \mathrm{C}\right){ }^{33,34}$ This broad agreement provides some confidence in the validity of the data analysis. (Of course, the friction in these experiments is between polar PVP units and units of butyl acrylate and acrylic acid and not between like units.)

Note that our argument presented here does not require the PVP chains to be fully miscible with the matrix. The theory for sliding friction at polymer/elastomer interfaces applies even when the pairs are weakly incompatible because of the intrinsic interpenetration width. ${ }^{15}$ Previous experimental work on the sliding friction of polymer melts has used end-grafted brush structures, ${ }^{13,14,15}$ but we expect friction effects at interfaces to be operative even if there is a loop-and-train structure of chains at the interface and limited miscibility with the continuous matrix. The basic principles are still valid.

The precise conformation of the PVP chains when adsorbed on the MWNTs is not known to us, but there are some recent coarse-grained Monte Carlo simulations ${ }^{35}$ that offer some needed insight. The simulation found that flexible chains (such as PVP) are less prone to helical adsorption (i.e. wrapping) in comparison to stiffer chains. Moreover, flexible chains show negligible overlap over the range of concentrations studied. The conformation of flexible chains adsorbed on larger radii was described as 
"cloud-like" These simulations suggest that there will be some extension of the PVP chains away from the carbon surface, and that they will not lie flat nor wrap.

A low interfacial strength will inhibit effective stress transfer. An improved interfacial strength will help to achieve full stress transfer from the matrix to the nanotube and will result in greater nanocomposite strength. Fig. 4 shows the interrelationship between the nanocomposite's engineering toughness (which is determined from the area under the stress-strain curve) and the $\tau$ deduced from the macroscopic strength, as already explained. The toughness enhancement is apparent in these soft nanocomposites. As the matrix macromolecules are in the rubbery state and have high mobility, an increased interfacial strength allows the nanocomposites to be deformed to a greater extent before fracture. The nanocomposites are therefore able to dissipate more energy, as previous results have revealed. ${ }^{5,17}$ A similar correlation between Young's modulus and $\tau$ is also shown. The nanocomposites exhibit greater stiffness when there is more contribution from the nanotubes as a result of increased stress transfer. Although, in principle, stress transfer can be achieved by covalent bonding between nanofillers and the matrix, ${ }^{36}$ our approach relies on a physical mechanism.

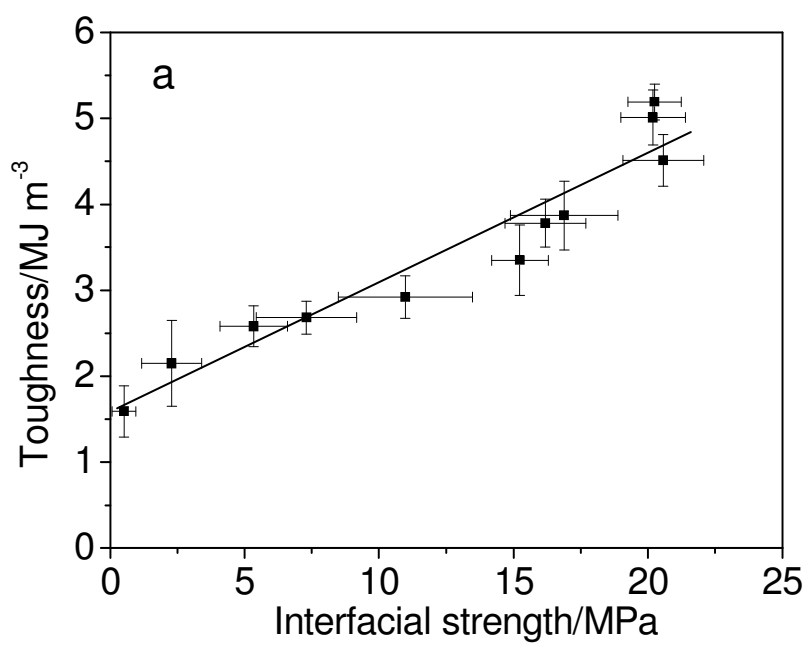






Figure 4. a) The correlation of macroscopic nanocomposite toughness with the interfacial strength, $\tau$.

b) The correlation of macroscopic nanocomposite Young's modulus with the interfacial strength. The solid line is the best linear fit to the data.

We note that $\bar{N}$ and $\Sigma$ are important parameters in the design of nanocomposites of this type. Their values can be adjusted to increase the macroscopic mechanical properties of the nanocomposite. Even when the molecular weight polydispersity is somewhat broad, as was the case here, $\bar{N}$ has a signficant impact on mechanical properties.

Our results also indicate that, in the limit of high velocity, the mechanical strength is a function of $\xi_{1}$. The value of $\xi_{1}$ can vary widely depending on the nature of the polymer, and hence the selection of the polymer dispersant on the nanotubes becomes an important design parameter. For instance, molecules with bulky and stiff repeat units typically have a $\xi_{1}$ value on the order of $\mu \mathrm{Nsm}^{-1}$, whereas others, e.g. polybutadiene, have values as low as a few $\mathrm{pNsm}^{-1}$. (33)

\section{Conclusions}

In a systematic study, we have demonstrated that the interfacial strength between carbon nanotubes and a soft polymer matrix increases with the chain length and density of polymer chains adsorbed at the interface. Longer chain lengths and higher chain densities both increase the interfacial strength and hence lead to an increased macroscopic strength (by a factor of two) and toughness (by a factor of three) 
Published in Macromolecules (2008) 41(20):7656-7661

in the nanocomposite. The observed increase in the interfacial strength is attributed to the sliding friction between the adsorbed dispersant chains and the matrix. These experiments thereby demonstrate a physical approach to improve the mechanical properties of nanocomposites, rather than one that relies on covalent bonding at the interfaces. The effect is quite forgiving of molecular weight polydispersity, and drawing from the results here, does not require monodisperse fractions.

The results lead us to propose that the monomer friction coefficient of the interfacial polymers is an important parameter that can be selected to tune the interfacial strength and hence the macroscale properties of nanocomposites of this type.

Acknowledgments. We thank Prof. J.M. Asua and M. Manea at the Institute for Polymer Materials (POLYMAT), The University of the Basque Country, Spain for providing the poly(BuA-co-AA) latex. We have benefitted from useful discussions with Prof. C. Creton (ESPCI, Paris) and Dr. R.P. Sear (Surrey). T.W. acknowledges an Overseas Research Student Scholarship from Universities UK and a University Research Scholarship from the University of Surrey.

Supporting information available: The effects of the free PVP chains, which were introduced in the nanocomposite preparation procedure, were investigated through tensile measurements and are shown in Figure S1. 
Published in Macromolecules (2008) 41(20):7656-7661

\section{References}

(1) Kim, J. K.; Mai, Y. W. Compos. Sci. Technol. 1991, 41, 333-378.

(2) Bonderer, L.J.; Studart, A. R.; Gauckler, L. J. Science 2008, 319, 1069-1073.

(3) Coleman, J. N.; Khan, U.; Blau, W. J.; Gun'ko, Y. K. Carbon 2006, 44,1624-1652.

(4) Callister, W. D. Materials Science and Engineering, An Introduction. $7^{\text {th }}$ ed. John Wiley \& Sons, Inc. 2007, p 585-595.

(5) Zhou, T. H.; Ruan, W. H.; Rong, M. Z.; Zhang, M. Q.; Mai, Y. L. Adv. Mater. 2007, 19, 26672671

(6) Shi, J. H.; Yang, B. X.; Pramoda, K. P.; Goh, S. H. Nanotechnology 2007, 18, 375704.

(7) Xie, L.; Xu, F.; Qiu, F.; Lu, H. B.; Yang, Y. L. Macromolecules 2007, 40, 3296-3305.

(8) Yerushalmi-Rozen, R.; Szleifer, I. Soft Matt. 2006, 2, 24-28.

(9) Creton, C.; Kramer, E. J.; Hui, C.-Y.; Brown, H. R. Macromolecules 1992, 25, 3075-3088.

(10) Norton, L. J.; Smigolova, V.; Pralle, M. U.; Hubenko, A.; Dai, K. H.; Kramer, E. J. Macromolecules 1995, 28, 1999-2008.

(11) Dai, C.-A.; Kramer, E. J.; Washiyama, J.; Hui, C.-Y. Macromolecules 1996, 29, 7536-7543.

(12) Creton, C.; Brown, H. R.; Shull, K. R. Macromolecules 1994, 27, 3174-3183.

(13) Brown, H.R. Science 1994, 263, 1411-13.

(14) Bureau, L.; Léger, L. Langmuir 2004, 20, 4523-4529.

(15) Casoli, A.; Brendlé, M.; Schultz, J.; Auroy, P.; Reiter, G. Langmuir 2001, 17, 388-398. 
Published in Macromolecules (2008) 41(20):7656-7661

(16) Wang, T.; Lei, C.-H.; Dalton, A. B.; Creton, C.; Lin, Y.; Fernando, K. A. S.; Sun, Y.-P.; Manea, M.; Asua, J. M.; Keddie, J. L. Adv. Mater. 2006, 18, 2730-2734.

(17) Wang, T.; Lei, C.-H.; Liu, D.; Manea, M.; Asua, J. M.; Creton, C.; Dalton, A. B.; Keddie, J. L. Adv. Mater. 2008, 20, 90-94.

(18) Cadek, M.; Coleman, J. N.; Ryan, K. P.; Nicolosi, V.; Bister, G.; Fonseca, A.; Nagy, J. B.; Szostak, K.; Beguin, F.; Blau, W. J. Nano Lett. 2004, 4, 353-356.

(19) Aradian, A.; Raphael, E.; de Gennes, P.-G. Macromolecules 2002, 35, 4036-4043.

(20) Wang, Y.; Winnik, M. A. J. Phys. Chem. 1993, 97, 2507-2515.

(21) Barber, A. H.; Cohen, S. R.; Wagner, H. D. Appl. Phys. Lett. 2003, 82, 4140-4142.

(22) Liao, K.; Li, S. Appl. Phys. Lett. 2001, 79, 4225-4227.

(23) Barber, A. H.; Cohen, S. R.; Eitan, A.; Schadler, L. S.; Wagner, H. D. Adv. Mater. 2006, 18, 8387.

(24) Jin, L.; Bower, C.; Zhou, O. Appl. Phys. Lett. 1997, 73, 1197-99.

(25) Dalmas, F.; Chazeau, L.; Gauthier, C.; Masenelli-Varlot, K.; Dendievel, R.; Cavaille, J.Y.; Forro, L. J. Polym. Sci. Pt. B. 2005, 43, 1186-97.

(26) Yang, S. G.; Zhang, Y. J.; Guan, Y.; Tan, S. X.; Xu, J.; Cheng, S. J.; Zhang, X. L. Soft Matt. 2006, 2, 699-704.

(27) Washiyama, J.; Kramer, E. J.; Creton, C.; Hui, C.-Y. Macromolecules 1994, 27, 2019-2024.

(28) Brown, H.R. Macromolecules 1991, 24, 2752-56.

(29) Yang, M.; Koutsos V.; Zaiser, M. J. Phys. Chem. B 2005, 109, 10009-14. 
Published in Macromolecules (2008) 41(20):7656-7661

(30) Israelachvili, J.N. Intermolecular and Surface Forces, 2nd ed. Academic Press 1992, p. 32.

(31) Rubinstein, M.; Ajdari, A.; Leibler, L.; Brochard-Wyart, F.; de Gennes, P. G. C. R. Acad. Sci. Paris, Ser. II 1993, 316, 317-320.

(32) Ajdari, A.; Brochard-Wyart, F.; de Gennes, P. G.; Leibler, L.; Viovy, J. L.; Rubinstein, M. Physica A 1994, 204, 17-39.

(33) Rodriguez, F.; Cohen, C.;. Ober, C. K.; Archer, L. A. Principles of Polymer Systems. 5th ed. Taylor \& Francis. 2003, p 258-259.

(34) Ferry, J. D. Viscoelastic properties of polymers 3rd ed. John Wiley \& Sons, Inc. 1980, p 328342.

(35) Gurevitch, I.; Srebnik, S. J. Chem. Phys. 2008, 128, 144901 (8 pages).

(36) Lin, Y.; Zhou, B.; Fernando, K. A. S.; Liu, P.; Allard, L. F.; Sun, Y.-P. Macromolecules 2003, $36,7199-7204$. 
Published in Macromolecules (2008) 41(20):7656-7661

Supporting Information

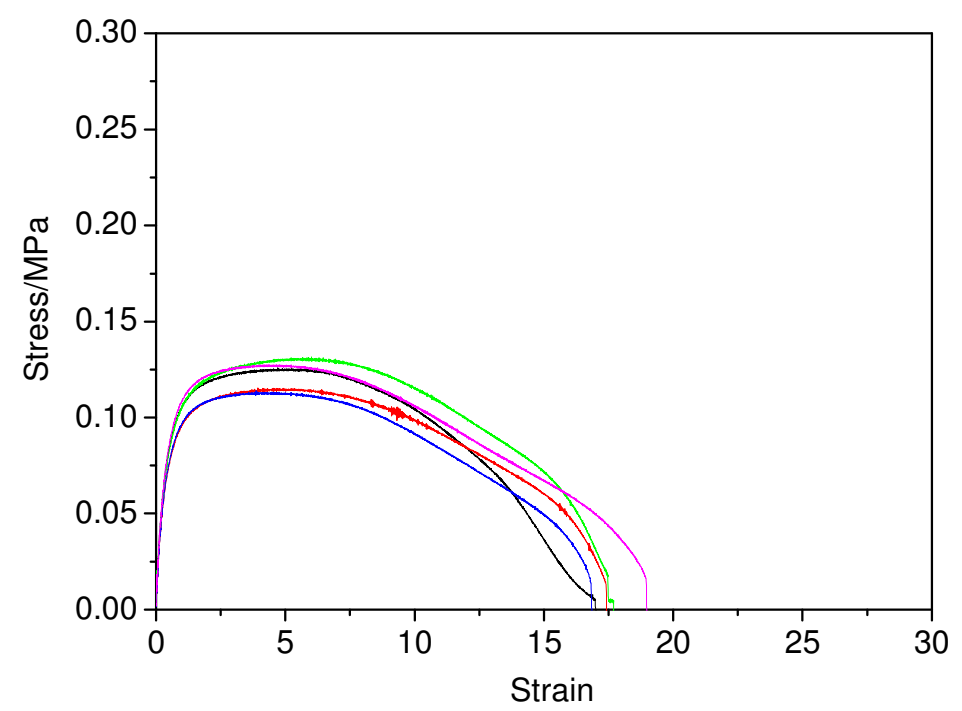

Fig S1: Large-strain tensile deformation of pure $\mathrm{P}(\mathrm{BuA}-\mathrm{co}-\mathrm{AA})$ and blends with $\mathrm{PVP}$ in which there is the same amount of free PVP as in the nanocomposites. Various PVP molecular weights and concentrations corresponding to various chain densities are compared: PVP $\bar{N}=14144$, $\Sigma=2.5 \times 10^{-3} \mathrm{~nm}^{-2}(-)$; PVP $\bar{N}=14144, \Sigma=4.5 \times 10^{-3} \mathrm{~nm}^{-2}(-) ; \operatorname{PVP} \bar{N}=90, \Sigma=0.157 \mathrm{~nm}^{-2}(-)$; PVP $\bar{N}=14144, \Sigma=0.01 \mathrm{~nm}^{-2}(-)$; and pure P(BuA-co-AA) film(-). The effects of the free PVP incorporated in the nanocomposites illustrate negligible mechanical impact within the experimental uncertainty. The axes are identical to those in Figures 2 and 3 to allow easy comparison. 
Published in Macromolecules (2008) 41(20):7656-7661

For Table of Contents use only

The importance of molecular friction in a soft polymer-nanotube nanocomposite

Tao Wang, Alan B. Dalton and Joseph L. Keddie

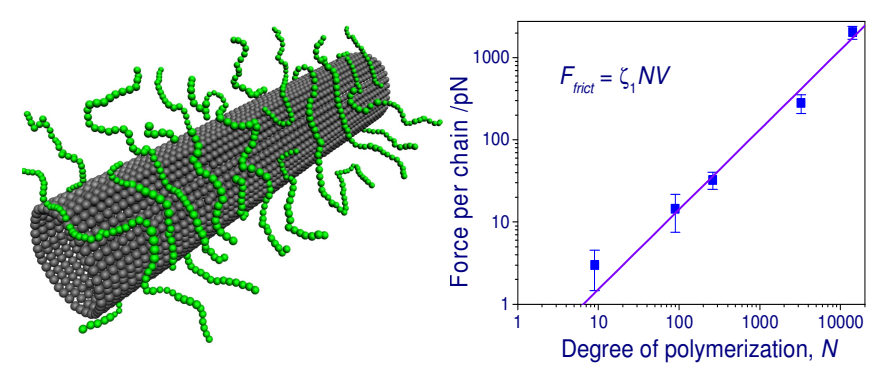

